\title{
Variación temporal de la concentración de monóxido de carbono usando datos del sensor MOPITT en las regiones costa, sierra y selva del Perú
}

Temporal variability of the carbon monoxide concentration using the MOPITT sensor's satellite data in Peru's coast, mountains and rainforest regions

Eduardo Ñaña Baquerizo

Universidad Continental

\section{RESUMEN}

Objetivos: Analizar la variabilidad temporal de la concentración del monóxido de carbono (CO) en las regiones costa (Chilca, Lima), sierra (Huancayo, Junín) y selva (Puerto Maldonado) para el período 2002-2012. Métodos: Investigación de nivel descriptivo. Se utilizaron datos satelitales generadas por el sensor MOPITT (measurements of pollution in the troposphere). Resultados: Se encontró que los datos del CO muestran un promedio de 2,67 $(1018 \mathrm{~mol} / \mathrm{cm} 2)$, en Chilca; $1,89(1018 \mathrm{~mol} /$ $\mathrm{cm} 2)$ en Huancayo y 4,70 (1018 $\mathrm{mol} / \mathrm{cm} 2)$ en Puerto Maldonado; los cuales fueron validados estadísticamente $(p>0,05)$, con valores máximos durante los meses de agosto a octubre y valores mínimos de mayo a julio, por un período de once años. Durante la investigación se registraron mayores concentraciones de $\mathrm{CO}$ en los años 2007 y 2010 y menores concentraciones de CO en los años 2006 y 2009. Se observó que en la concentración de $\mathrm{CO}$, existe un descenso en Chilca y Puerto Maldonado de $-0,52 \%$ /año y $-0,60 \%$ /año, respectivamente, y un incremento en Huancayo de $0,05 \%$ /año. Los valores en Chilca tienen máximos de 2,12 en setiembre y 2,03 en octubre; en Puerto Maldonado tienen máximos de 3,23 en setiembre y 2,98 en octubre; en Huancayo tienen máximos de 1,27 en setiembre y 1,22 en octubre. Conclusiones: Los hallazgos evidencian un patrón estacional en la concentración de $\mathrm{CO}$ que se mantiene para todos los años sobre las tres ciudades, con un incremento de agosto a octubre, resultando Puerto Maldonado la ciudad con mayor concentración de $\mathrm{CO}$ y Huancayo con menor concentración.

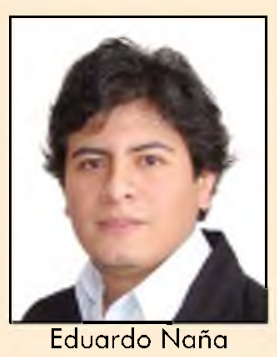

Baquerizo.ee@gmail.com

Historial del artículo: Recibido: 3 de mayo de 2014 Aprobado: 17 de noviembre de 2014 Disponible en línea: 30 de diciembre de 2014

Palabras clave: Variación temporal, concentración monóxido de carbono, sensor MOPITT. 


\section{ABSTRACT}

Objectives: To analyze the temporal variability of the carbon monoxide concentration (CO) in Peru's coast (Chilca, Lima), mountains (Huancayo, Junin) and rainforest (Puerto Maldonado) regions for the period 2002-2012. Methods: Descriptive level research. MOPITT sensor's satellite data were used (pollution measurements in the troposphere). Results: We found that CO data show an average of 2,67 (1018 $\mathrm{mol} / \mathrm{cm} 2)$, in Chilca; $1,89(1018 \mathrm{~mol} / \mathrm{cm} 2)$ in Huancayo and $4,70(1018 \mathrm{~mol} / \mathrm{cm} 2)$ in Puerto Maldonado; which were statistically validated $(p>0,05)$, with maximum values from august to october and minimum values from may to july, for a period of eleven years. During the research, higher $\mathrm{CO}$ concentrations were recorded in 2007 and 2010 and lower $\mathrm{CO}$ concentrations in 2006 and 2009. It was observed that in the $\mathrm{CO}$ concentration, there is a decrease in Chilca and Puerto Maldonado of $-0,52 \% /$ year and $-0,60 \% / y e a r$, respectively, and an increase in Huancayo of $0,05 \% /$ year. The values in Chilca are maximum of 2,12 in september and 2,03 in october; in Puerto Maldonado are maximum of 3,23 in september and 2,98 in october; in Huancayo are maximum of 1,27 in september and 1,22 in october. Conclusions: The findings show a seasonal pattern in the $\mathrm{CO}$ concentration, which is maintained for all the years on the three cities, with an increase from august to october, Puerto Maldonado is the city with the highest $\mathrm{CO}$ concentration and Huancayo with the lower $\mathrm{CO}$ concentration.

Keywords: Temporal variability, carbon monoxide concentration, MOPITT sensor.

\section{INTRODUCCIÓN}

El impacto de la contaminación atmosférica global sobre el clima y el ambiente es un nuevo enfoque en la ciencia atmosférica (1). La importancia de estos contaminantes gaseosos se debe a que pueden ser transportados a grandes distancias de sus fuentes, $y$ en determinadas circunstancias tienen efectos sobre la calidad del aire local. Siendo uno de los principales contaminantes, el monóxido de carbono (CO) que es un marcador útil para otros contaminantes, como el ozono troposférico. La vida atmosférica de $\mathrm{CO}$ es de unas pocas semanas a varios meses en la atmósfera, lo que le permite viajar largas distancias y generar impactos en la calidad del aire (2).

El CO es liberado a la atmósfera por una variedad de actividades de combustión incompleta, incluyendo la quema de gas natural, combustibles fósiles y otros combustibles que contienen carbono (C) con un importante impacto en la química de la atmósfera a través de su reacción con hidroxilo $(\mathrm{OH})$ en la formación de ozono en la tropósfera (3). Además, incluso a niveles bajos de concentración es peligroso para la vida humana por lo tanto, debe ser monitorizada exacta y precisa en tiempo real. (4)

En Sudamérica, la quema de biomasa contribuye de manera importante a la contaminación del aire con partículas y gases de invernadero en todo el mundo. Siendo la Amazonía una zona de abundante actividad por quemas de vegetación, como parte del cambio de uso de suelos para aprovechamiento agrícola (5). Estas quemas se presentan principalmente en la época seca (de mayo a octubre), que también coincide con la menor capacidad de remoción de los contaminantes por las mínimas lluvias y con ello una mayor posibilidad de que los contaminantes alcancen mayores distancias.

Son escasos los estudios relacionados a la variabilidad temporal del $\mathrm{CO}$ a nivel mundial. En Sudamérica se viene investigando en el impacto de las quemas de biomasa y de otros factores que influyen en la producción de contaminantes atmosféricos.

Estudios realizados en América del norte utilizaron datos MOPITT para examinar el transporte y variación del $\mathrm{CO}$ en el hemisferio sur $y$ en el hemisferio Norte (6). Obtuvieron como resultados que la variación estacional de $\mathrm{CO}$ global en 
general muestra dos picos en abril-mayo y octubre-noviembre

En el estudio realizado en Brasil analizaron los efectos de la contaminación del aire en las enfermedades cardiovasculares en la ciudad de Sao José dos Campos en Sao Paulo (7). El estudio encontró que el número de enfermedades están relacionadas con la concentración del contaminante $\mathrm{CO}$ y los factores meteorológicos, los cuales se dan en los meses de julio, agosto y septiembre.

Como se mencionó antes, la relación de la quema de biomasa y contaminantes atmosféricos es alta, por ello en el estudio de Torres 0 , et al. (8); analizaron la temporada de incendios en América del Sur y África Central durante los últimos diez años para entender los factores que llevaron a los extremos de 2007 y 2009, por medio de los sensores OMI y MODIS.

En otro trabajo (9) exploraron las relaciones espaciales y temporales entre $\mathrm{CO}$, aerosoles y la quema de vegetación en el norte de argentina a través de imágenes y datos satelitales así como cálculo de trayectorias y análisis de situaciones meteorológicas. Argumentaron que los valores máximos del $\mathrm{CO}$ se da de julio a octubre, producto de las emisiones de la quema de biomasa transportados desde Brasil, Bolivia y Paraguay.

Para mejorar el uso de la teledetección en el monitoreo atmosférico este trabajo se ha planteado el siguiente problema ¿Cómo es la variabilidad temporal de los datos de concentración del monóxido de carbono (CO) sobre Chilca, Huancayo y Puerto Maldonado? El objetivo fue identificar la variabilidad estacional de la concentración del CO sobre las ciudades de Chilca, Huancayo y Puerto Maldonado en base a los datos de Columna total registrados por el sensor MOPITT para el periodo 20022012.

La presente investigación se plantea como hipótesis, que la variabilidad estacional de los datos de concentración de $\mathrm{CO}$ y NO2 sobre Chilca, Huancayo y Puerto
Maldonado muestra valores trimestrales máximos en los meses de agosto a octubre y mínimos en los meses de Mayo a Julio.

En el Perú, aún no se ha realizado estudios relacionados a la variabilidad de la concentración del $\mathrm{CO}$ a través de datos satelitales del sensor MOPITT. Se espera, por medio de este trabajo, dar soporte en el estudio de medición remota de este contaminante. Puesto que será una herramienta importante emplear la teledetección para los sistemas de gestión de la calidad del aire y posteriormente ser utilizados por los organismos encargados de controlar la contaminación atmosférica.

En el transcurso de la investigación se tuvo inconvenientes con la obtención de los datos por problemas técnicos del portal NCAR Earth System Laboratory diseñado por la National Aeronautics and Space Administration (NASA). A ello se suma las dificultades en el entendimiento $y$ procesamiento de los datos satelitales.

Para una mejor comprensión del $\mathrm{CO}$ se requiere de mayor investigación con temas relacionados a la variabilidad del $\mathrm{CO}$ como, el impacto del transporte transfronterizo de contaminantes producto de las quemas de biomasa, análisis climatológicos como la precipitación, dirección y velocidad de viento, por medio de monitoreo in-situ y de trabajos de modelamiento.

\section{MATERIAL Y MÉTODOS}

El tipo de investigación propuesta fue básica, el nivel de investigación es descriptivo, y el tipo de diseño de investigación es longitudinal. Para realizar una mejor caracterización del comportamiento de la concentración del $\mathrm{CO}$, se analizó la variabilidad interanual, mensual y trimestral sobre las ciudades de Chilca (Lima, región costa), Huancayo (Junín, región sierra) y Puerto Maldonado (Madre de Dios, región selva). La ubicación de estos sitios se detalla en la tabla 1.

La concentración de CO, fue generado por el 
Tabla $N^{\circ}$ 1: Ubicación geográfica de los puntos que comprenden el área inicial de estudio.

\begin{tabular}{lcc}
\hline \multicolumn{1}{c}{ Ciudad } & Latitud & Longitud \\
\hline Chilca (costa) & $-12,50$ & $-76,80$ \\
Huancayo (sierra) & $-12,05$ & $-75,32$ \\
$\begin{array}{l}\text { Puerto Maldonado } \\
\text { (selva) }\end{array}$ & $-12,57$ & $-70,10$ \\
\hline
\end{tabular}

MOPITT (Measurements Of Pollution In The Troposphere) instalado a bordo del satélite TERRA (Figura 2). El instrumento MOPITT es un infrarrojo térmico multicanal (TIR) e infrarrojo cercano (NIR), a bordo del satélite EOS instrumento -Terra. MOPITT tiene una resolución espacial horizontal a $22 \mathrm{~km} \times 22$ $\mathrm{km}$ y ancho de franja unos $640 \mathrm{~km}$, lo que permite una cobertura global cada 3 días. MOPITT utiliza radiometría correlación de células de gas para detectar la absorción de CO atmosférico de 4,6 micrómetros (canales TIR) y 2,3 micrómetros (canales NIR), (10, 11). Los perfiles se recuperan en 7 niveles verticales, desde la superficie hasta $100 \mathrm{hPa}$ utilizando un método de estimación óptima no lineal. Los algoritmos de recuperación de MOPITT V6 contienen mejoras de las deficiencias de versiones anteriores, una mayor resolución espacial $10,66^{\circ} \times 0,5^{\circ}$ vs $1^{\circ} \times 1^{\circ}$ ), y mejor geolocalización (latitud $y$ longitud), mediante la corrección de la tabla en el procesador de Nivel 1, que define la pista cruzada y ángulos de visión a lo largo de la pista para cada posición de píxel (11).

El algoritmo de recuperación MOPITT requiere perfiles de temperatura y vapor de agua, así como a priori los valores de temperatura de la superficie, por ello mejora los campos climatológicos. En los procesadores operativos anteriores, los datos meteorológicos se obtuvieron de NCEP GDAS (Global Data Assimilation System) los productos de análisis. Para el procesamiento de V6, los perfiles meteorológicos se derivan de la NASA MERRA (Modern-Era Retrospective Analysis For Research And Applications), obteniendo como resultado una mayor calidad de los datos recuperados por dicho sensor (12). La fecha de registro MOPITT cubre marzo de 2000 hasta el presente y los productos con los que cuenta el sensor son: CO Profile Mixing Ratio, Retrieved CO total Column, $\mathrm{CH} 4$ Total Column Abundance y Radiance.

En este estudio se utilizó el producto "Retrieved CO total Column" procedentes del sensor MOPITT, Los datos fueron tomados de día con cielo despejado, derivados de las bandas TIR/NIR para el periodo 20022012. La data fue proporcionada por el Dr. Merrit Deeter líder del proyecto MOPITT y de la Investigadora Sara Martínez Alonso.

Las técnicas de procesamiento estuvieron basadas en el uso de MS Excel, Matlab y Statgraphics. Para el tratamiento de datos se aplicará la estadística descriptiva (medidas de tendencia central y dispersión) e inferencial (Prueba de Kruskal-Wallis)

\section{RESULTADOS}

Durante todo el periodo de recolección de datos (2002-2012) se obtuvo valores máximos y mínimos de 2,67 y 1,30 en Chilca (costa); 1,90 y 0,83 en Huancayo (sierra) y en Puerto Maldonado (selva) los valores más extremos de 4,70 y 1,31 . Se registró un promedio de 1,74, 0,83 y 1,92 para Chilca (costa), Huancayo (sierra) y Puerto Maldonado (selva) respectivamente. Del mismo modo se comparó la media con la mediana sin obtener mucha variación, en Chilca (costa) se observó una media de 1,74 y una mediana de 1,71 , en Huancayo (sierra) una media de 1,07 y una mediana de 1,03 y en Puerto Maldonado (selva) una media de 1,92 y una mediana de 1,72. Y con una desviación estándar de $\pm 0,24$, $\pm 0,19$ y $\pm 0,61$ en costa, sierra y selva respectivamente.

\section{Variación interanual}

La evolución interanual de la concentración de $\mathrm{CO}$ en las tres ciudades está definida por dos patrones característicos: (1) máximos valores muy pronunciados y prolongados comprendidos entre finales de invierno y principios de primavera y (2) mínimos valores estivales. Los años 2005, 2007 y 2010 tienen mayor concentración de CO. 
La mayor concentración de contaminantes se encuentran en los meses de agosto, septiembre y octubre con un promedio durante estos meses de 1,20, 2,61 y 1,94 (1018 mol/cm2), sobre Chilca, Huancayo y Puerto Maldonado respectivamente. Los valores más bajos se observaron en los meses de lluvia, sobre todo de mayo a julio, con picos de incidencia en Octubre del 2007 de 4,074 (1018 mol/cm2) sobre Puerto Maldonado.

La concentración del $\mathrm{CO}$ en Huancayo se ha venido incrementando desde el 2002 hasta el 2012, de acuerdo con la regresión lineal los niveles de concentración del $\mathrm{CO}$ en Huancayo tienen un incremento de 0,0038 (1018 mol/cm2)/año $(p>0,05), y$

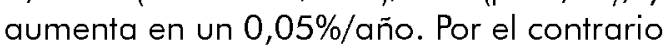
se observó un descenso de concentración del $\mathrm{CO}$ sobre Chilca y Puerto Maldonado de $-0,006$ y $-0,0053(1018 \mathrm{~mol} / \mathrm{cm} 2) / a n ̃ o$ $(p>0,05)$. $Y$ disminuyen en un $-0,52 \%$ y $-0,60 \%$ /año respectivamente.

\section{Variación mensual}

La figura $\mathrm{N}^{\circ} 01$, muestra la variabilidad mensual sobre las tres ciudades. Se observa que la concentración de $\mathrm{CO}$ mensualmente está diferenciada por dos períodos, En Puerto Maldonado durante el mes de mayo se registra el valor menor. En septiembre se registra la máxima concentración de
CO de todo el año alcanzando. En Chilca, se observó el mismo patrón de variación, con un valor máximo en agosto a octubre, observando un pico en setiembre y valores mínimos de mayo a julio. La variabilidad mensual sobre Huancayo se registró valor mínimo en junio y el valor máximo en setiembre.

\section{Variación trimestral}

La figura $\mathrm{N}^{\circ}$ 02, muestra la variación trimestral en Chilca, Huancayo y Puerto Maldonado. Se observa presentan valores máximos de $\mathrm{CO}$ en el trimestre agostosetiembre-octubre (ASO) (40\%) en selva y más del $25 \%$ sobre la costa y sierra. Por lo contrario se registró valores mínimos de $18 \%$ en los trimestres febrero-marzoabril (FMA) y de $16 \%$ en los trimestres noviembre-diciembre-enero (NDE).

\section{Variación estacional}

La figura $\mathrm{N}^{\circ}$ 03, la presencia de $\mathrm{CO}$ en las ciudades de Chilca, Huancayo y Puerto Maldonado, produce valores máximos y extremos en las estaciones de invierno y primavera, y valores mínimos en las estaciones de verano y otoño. Así, encontramos que sobre las tres ciudades se registró los valores más altos en primavera de $1,88 \pm 0,38,1,53 \pm 0,46$ y $2,71 \pm 1,14$

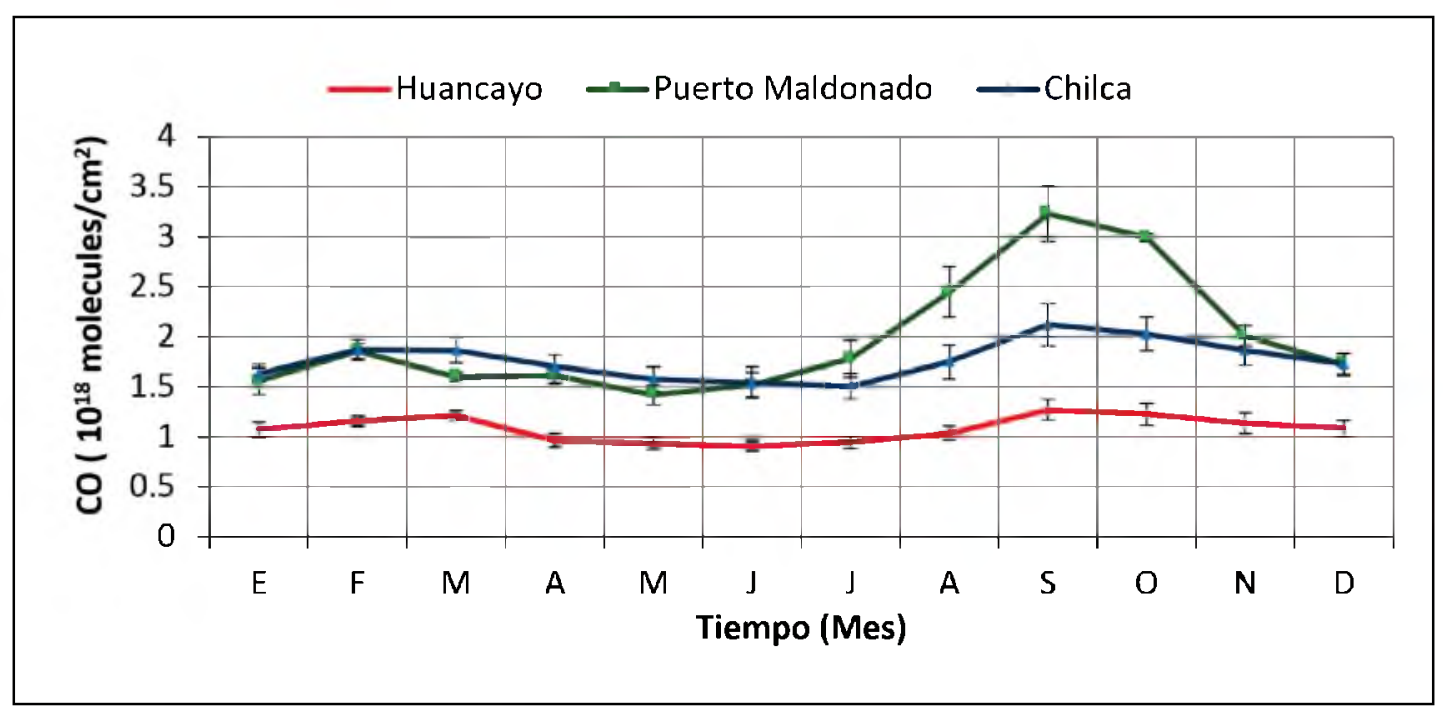

Figura $\mathrm{N}^{\circ}$ 1: Variabilidad mensual de la concentración de $\mathrm{CO}$ sobre Chilca, Huancayo y Puerto Maldonado, obtenida a partir del promedio. . 


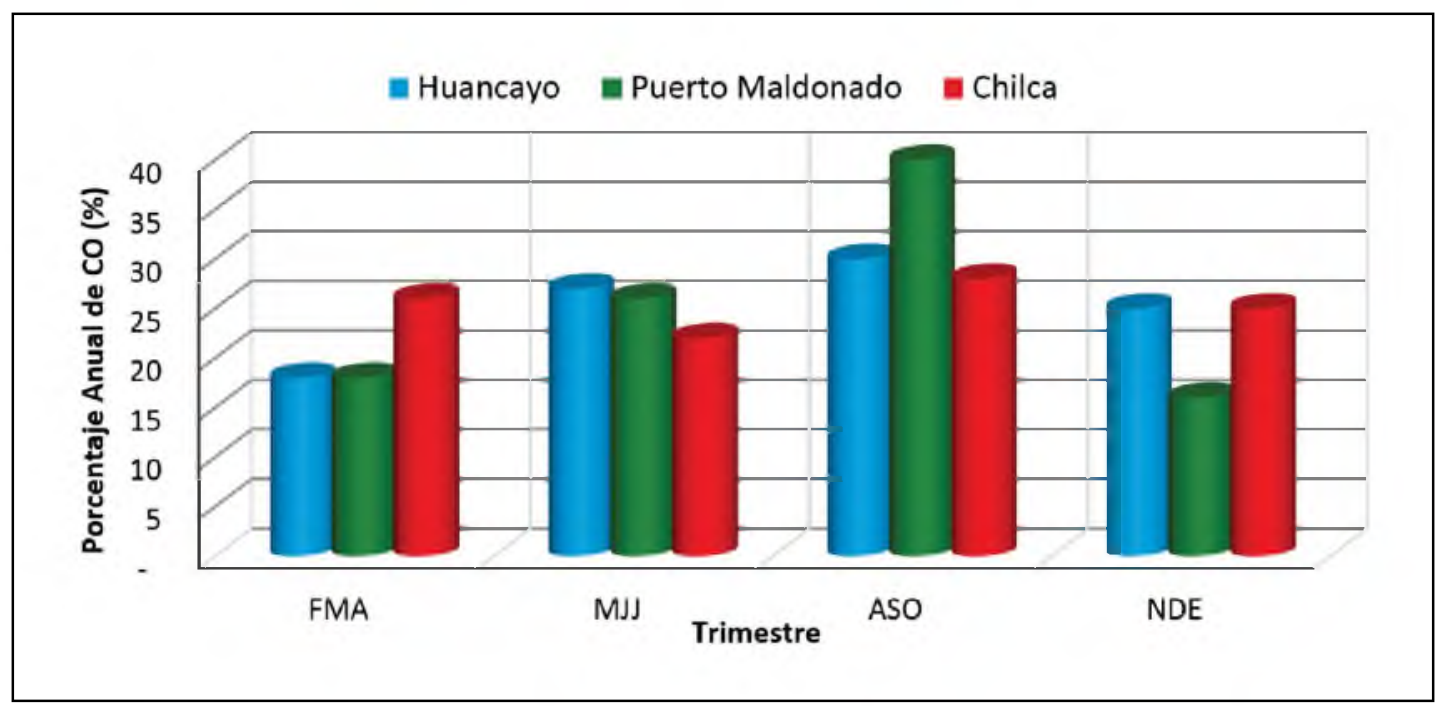

Figura $N^{\circ}$ 2: Porcentaje de CO por trimestres en Chilca-Lima, Huancayo y Puerto Maldonado para el período 2002-2012.

para Chilca, Huancayo y Puerto Maldonado respectivamente. De igual manera se registró los valores mínimos en otoño de $1,23 \pm 0,29$ y $1,55 \pm 0,27$ en Huancayo y Puerto Maldonado respectivamente, por el contrario en Chilca se registró el valor mínimo en la verano.

\section{DISCUSIÓN}

En base al comportamiento de la concentración del $\mathrm{CO}$, se pudo observar que los valores de concentración de $\mathrm{CO}$ en las tres ciudades son todas diferentes, estos resultados se validó utilizando la prueba estadística no paramétrica Kruskal-Wallis, se escogió esta prueba debido a que los datos en Chilca, Huancayo y Puerto Maldonado no presentan una distribuciones normal. El resultado de la prueba fue que el valor-p es menor que 0,05 , con lo que se concluye que existe una diferencia estadísticamente significativa entre las medianas con un nivel del 95,0\% de confianza.

En la variabilidad anual se pudo observar que en el 2007 presenta valores máximos de $2,80 \pm 1,53(1018 \mathrm{~mol} / \mathrm{cm} 2$; desvt.

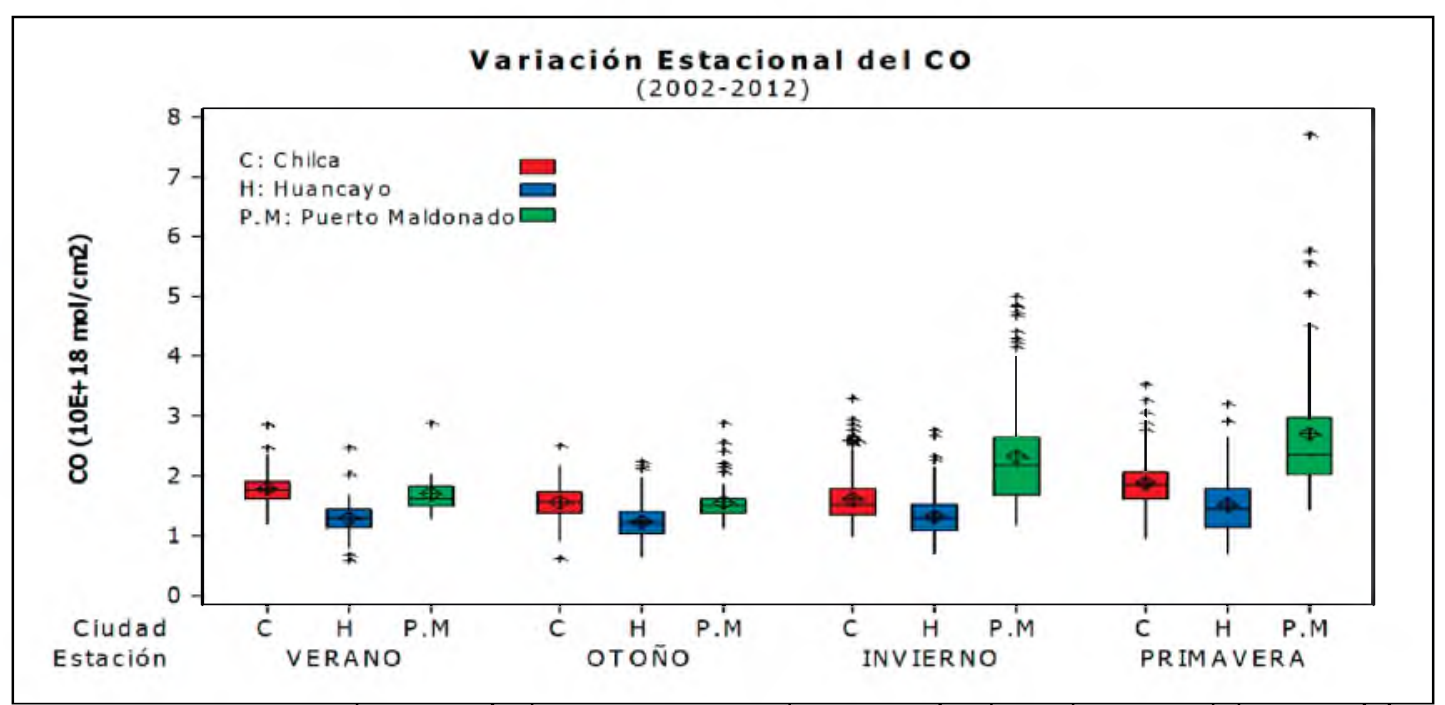

Figura $N^{\circ}$ 3: Diagrama de cajas de la concentración de monóxido de carbono medidos en Chilca, Huancayo y Puerto Maldonado durante el período 2002-2012. 
Std.), este valor se atribuye al aumento significativo de números de incendios que se observó en América del Sur uno de los mayores en los últimos diez años en la investigación de Torres $O$. et al. (8). $Y$ valores mínimos en el 2006 de 1,91 \pm 0,82 y $20091,58 \pm 0,24$, estos valores coincide con el menor número de incendios en América del Sur, este descenso se asocia con la implementación de una política de prevención de la quema de biomasa trinacional que participaron los gobiernos de Brasil, Bolivia y Perú (13).

La variación mensual de $\mathrm{CO}$ es impulsada por factores como el ciclo estacional de $\mathrm{OH}$, la ubicación y la distribución de sus fuentes (3). Por ello los ciclos estacionales observados en sobre Puerto Maldonado, presentan una exposición de ciclo mucho más fuerte con valores altos de 3,23 (setiembre) debido a que Puerto Maldonado se encuentra en una región que experimenta altas concentraciones de $\mathrm{CO}$ (fuentes de contaminación). Esto conduce a un rápido aumento en las concentraciones en septiembre, cuando las altas concentraciones de $\mathrm{CO}$ de las fuentes que provienen de Brasil son transportados a este ubicación remota.

Los ciclos mensuales de las ciudades Chilca y Huancayo presentan una exhibición a una escala pequeña, con valores máximos en setiembre de 2,12 y 1,27 respectivamente. Esta variabilidad es causada por el transporte a larga distancia de $\mathrm{CO}$ sobre estas ciudades procedentes de fuentes alejadas de quemas de biomasa del Brasil, Perú y Paraguay (9).

Varios estudios dan cuenta de la periodicidad del CO, por ejemplo Moura, et al. (7) obtuvieron como resultado para el periodo 2000-2007, mayores concentraciones en los meses de agosto, septiembre y octubre $(1,7 ; 2,3 ; 2,1 \times$ $1018 \mathrm{~mol} / \mathrm{cm}^{2}$, respectivamente). Siendo similares a los resultados arrojados en el estudio, esto debido a que en el hemisferio sur tiene esta estacionalidad y con picos en estos meses. El mismo caso se observó en la investigación de Drummond J. et al
(8), registraron valores máximos en agosto, setiembre y octubre para el hemisferio sur y marzo, abril y mayo para el hemisferio norte durante los años 2001 al 2003.

Con respecto a los resultados de variación trimestral y estacional se observó que en los meses y estaciones de baja concentración del $\mathrm{CO}$ se debe al incremento de precipitaciones en estos lugares, ya que estos producen radicales $\mathrm{OH}$ los cuales limpian la atmósfera para disminuir la cantidad de $\mathrm{CO}(4)$.

En Huancayo este fenómeno meteorológico es evidente, este mismo patrón se registró con menores valores en Chilca y Puerto Maldonado (15), por ello en Huancayo se registró los valores mínimos de $\mathrm{CO}$ en el trimestres (FMA) y valores máximos en el trimestre (ASO). Debido a que durante el año se presenta dos épocas muy típicas un periodo seco (estiaje), comprendido entre los meses de abril-setiembre y otra estación húmeda (lluvioso), que comprende los meses de octubre-marzo como reporta Arroyo J. (16)

El CO tiene una relación directa con el ozono troposférico (O3), que se registró más del $30 \%$ del 03 anual en los meses de agosto-septiembre-octubre en el trabajo de Velarde F. (17), siendo los mismos meses que se observó valores máximos de $\mathrm{CO}(40$ $\%$ ) en la presente investigación. Con lo que se reafirma que el $\mathrm{CO}$ es un precursor en la formación del $\mathrm{O}$.

Los datos recolectados y procesados por el sensor MOPITT permitieron cuantificar adecuadamente la concentración de $\mathrm{CO}$ en las ciudades de Chilca, Huancayo y Puerto Maldonado. Las tres ciudades seleccionadas exhiben ciclos estacionales que siguen el mismo patrón amplio. Las concentraciones de $\mathrm{CO}$ poseen valores mínimos durante en los meses de verano. El nivel de CO luego aumenta hasta el otoño y el invierno, ya que las concentraciones de $\mathrm{OH}$ disminuyen, hasta un máximo en primavera, siendo Puerto Maldonado la ciudad con mayor concentración de $\mathrm{CO}$ y Huancayo la ciudad con menor concentración de $\mathrm{CO}$. 
Esta variación se debe a fuentes como la quema de biomasa y factores climatológicos (precipitación y dirección del viento).

\section{Agradecimiento}

A la United States Agency for International Development (USAID), National Academy of Sciences (NAS) por el financiamiento del proyecto, al Dr. Deeter Merrit, líder del proyecto MOPITT y a la Investigadora Sara Martínez Alonzo, por el apoyo en la obtención de los datos satelitales.

\section{REFERENCIAS BIBLIOGRÁFICAS}

1. Ramanathan $V$, Feng $Y$. Air pollution, greenhouse gases and climate change: Global and regional perspectives. Atmospheric Environment [Internet]. 2009 Enero [Citado el 20 de marzo de 2014]; 43(1): 37-50. Disponible en: http://ubairpollution. org/Papers/General_and_Review/ Ramanathan_2009.pdf

2. Emmons $L$, Deeter $M$, Gille J, Edwards D, Attié J, Warner J, et al. Validation of measurements of pollution in the troposphere (MOPITT) CO retrievals with aircraft in situ profiles. Journal of Geophysical Research. 2004; 109(D3): 1-13.

3. Spivakovsky C, Logan J, Montzka S, Balkanski $Y$, Foreman $M$, Jones $D$, ef al. Three-dimensional climatological distribution of tropospheric $\mathrm{OH}$ : Update and evaluation, J. Geophys. Res. 2000; 105(D7): 8931-8980.

4. Khalil M, Rasmussen R. Carbon monoxide in the earth's atmosphere: increasing trend. Science. 1984; 224(4644): 54-56.

5. Setzer A, Pereira M. Amazonia biomass burnings in 1987 and an estimate of their tropospheric emissions. AMBIO [Internet]. 1991 Febrero [Citado el 09 de marzo de 2014]; 20(1): 1922. Disponible en:http://queimadas. cptec.inpe.br/ rqueimadas/ documentos/1991_Setzer_Pereira_
Amazonia_AMBIO.pdf

6. Drummond J, Liu J, Nichitiu F, Kar $J$, Bremer H, Zou J, et al. Global Distributions of Carbon Monoxide Total Column: A Statistical Analysis from MOPITT Data. American Geophysical Union; 2004.

7. Moura $Y$, Pereira G, Moraes E, Arai $E$, Pereira C. Distribuição de CO no município de São José dos Campos a partir de dados do sensor MOPITT e sua relação com doenças cardiovasculares. Simpósio Brasileiro de Sensoriamento Remoto INPE [Internet], 2009 Diciembre [citado el 20 de Agosto de 2014]; 6697-6702. Disponible en: http://marte.sid.inpe.br/col/dpi.inpe. br/sbsr@80/2008/11.18.01.26/ doc/6697-6702.pdf

8. Torres $O$, Chen $Z$, Jethva $H$, Ahn $C$, Freitas $S$, Bhartia $P$ OMI and MODIS observations of the anomalous 20082009 Southern Hemisphere biomass burning seasons. Atmos. Chem. Phys. 2010; (10): 3505-3513.

9. Mielnicki D, Canziani P, Drummond J. Quema de biomasa en el centro-sur de Sudamérica: incendios locales, impactos regionales. Congreso Argentino de Meteorología. Buenos Aires; 2005. Disponible en: http://www.uca.edu.ar/ uca/common/grupo72/files/QUema_ biomasa_centro_sur_sudamerica.pdf

10. Deeter $\bar{M}$, Emmons $L$, Francis $G$, Edwards D, Gille J, Warner J, et al. Operational carbon monoxide retrieval algorithm and selected results for the MOPITT instrument, J. Geophys. Res. 2003; 108(D14): 1-11.

11. Drummond J, Zou J, Nichitiu F, Kar J, Deschambaut R, Hackett J. A review of 9-year performance and operation of the MOPITT instrument. Adv. Space. Res. [Internet]. 2010 Marzo [Citado el 12 de marzo de 2014]; 45: 760774. Disponible en: http://www. sciencedirect.com/science/article/pii/ S0273117709007224\#

12. Deeter M. MOPITT Version 6 Product User's Guide. Boulder: National Center for Atmospheric Research; 2013. Disponible en: https://www2.acd.ucar. edu/sites/default/files/mopitt/v6_users_ 
guide_201309.pdf

13. Schroeder W, Giglio L, Aravéquia J, Comment on "Reversal of trend of biomass burning in the Amazon" by llan Koren, Lorraine A. Remer, and Karla Longo. Geophys. Res. Lett. [Internet]. 2009 Febrero [Citado el 19 de marzo de 2014]; 36(03): 1-4.

14. Novelli P, Masarie K. and Lang P Distributions and recent changes of carbon monoxide in the lower troposphere. J. Geophys. Res. 1998; 103(D15): 15-33.

15. Servicio Nacional de Meteorología e Hidrología del Perú. Normales decadales de temperaturas y precipitación y calendario de siembras y cosechas. Lima: Ministerio de Agricultura y Riego/ Corporación Gráfica Rodríguez SAC; 2013.

16. Arroyo J. Tendencias y cambio del clima del valle del Mantaro mediante los análisis de índices de Precipitación Efectiva y Temperatura Eficiente. Apunt. cienc. soc. 2011 ; 01(01): 45-54.

17. Velarde F. Estudio de la variación estacional de aerosoles transportados a la ciudad de La Paz y su relación con las quemas de Biomasa. [Tesis doctoral]. La Paz: Universidad Mayor de San Andrés; 2010. 